$\mathbb{T}$ periodica polytechnica

Mechanical Engineering

$5 2 / 1 ( 2 0 0 8 ) \longdiv { 1 9 } 2 3$

doi: 10.3311/pp.me.2008-1.04

web: http://www.pp.bme.hu/me

(c) Periodica Polytechnica 2008

RESEARCH ARTICLE

\section{A transformation technique for efficient shape optimization in CAD/CAE environment}

\author{
Gábor Körtélyesi / Csilla Erdős-Sélley
}

Received 2008-01-18

\begin{abstract}
In the course of shape optimization in an integrated $C A D / F E M$ environment, finding of appropriate bounds of the shape parameters, has a principal importance: on one hand, non-recoverable geometries and structural model are to avoid, on the other hand, valid combination of shape parameters must not be excluded because they may include the optimum. To resolve this contradiction, a transformation is introduced to project the variable dimensions of the geometry onto the $n$ dimensional unit cube, so these normed design variables are used in the course of the optimization. In simpler cases this transformation can be derived from the limit relations of the geometry construction as it is shown on the optimization example of a handhold used in public transport.
\end{abstract}

\section{Keywords}

CAD-based shape optimization · optimization model

\section{Gábor Körtélyesi}

Department of Machine and Product Design, BME, 1111 Budapest Múegyetem rkp. 3., Hungary

e-mail: korte@eik.bme.hu

\section{Csilla Erdős-Sélley}

Department of Machine and Product Design, BME, 1111 Budapest Múegyetem rkp. 3., Hungary

e-mail: selley@eik.bme.hu

\section{Introduction}

Earlier on, shape optimization in the machine element design could be performed mainly for structural components with simple geometry by the programs available: optimization was performed by changing a few size parameters or the node coordinates of the structural model [1,2].

Newly there are a large number of methods and techniques for solving a variety of shape optimization problem [3].

FE model based optimization allows for changing the geometry of the body inspected only in smaller steps as a result of mesh changes, even by applying adaptive mesh technique [4]. By the integration of parametric CAD systems and FE systems with an automatic mesh generator the design variables can be altered in a wide range in a single step as the geometrical and structural model are rebuilt in each step. Another advantage of applying CAD dimension related design variables is, their impact can be exerted not only in a small local area of the body, but they can change the geometry of the body even globally [5]. Definition of the optimization model is much faster so bodies with complex geometry can also be studied in a short time. The great geometrical changes can result topological changes, complicating the task of preserving design goals [6]. Our purpose is to study the impact of formulation of the optimization domain. Until now this topic was not studied intensively, only some aspects of it as the design model refinement was studied [7]. Having shape optimization problems with more complex geometry to vary, the designer has to give a good answer to this question, independent from the examined problem, its type of modelling or the solution method.

\section{Definition of the optimization domain}

The surface of the inspected body can be drawn in a parametric CAD modeller by splines or other analytical functions, and it can be made fully defined by assigning dimensions to it. Design variables are chosen among these dimensions, having constant limits given by the designer. It means, the optimization domain is a rectangle in the $n$ dimensional Euclidean space where $n$ is the number of the design variables. The question arises what constants are needed to formulate an appropriate op- 
timization domain. These constants should be chosen on such a way that all points of the optimization domain are valid, namely the geometrical and the structural model can be rebuild. If the topology of the geometry (the number of the surfaces and the surface connection schema) is the same as by the initial design, the above-mentioned two conditions for feasibility are fulfilled. The domain of feasible points called regeneration domain. In order to have a valid design, the optimization domain must be a closed subset of the regeneration domain. However, the regeneration domain is not necessarily a rectangle and therefore a great amount of possible geometry is excluded from the optimization, probably also the optimum itself. To overcome this contradiction a mapping of the regeneration domain onto the rectangular domain is proposed. From computing aspects the normed design variables are advantageously used too. Therefore we have to find a mapping of the regeneration domain onto the unit cube of the same order. Although such a transformation is a very powerful tool, it cannot be given generally for all kinds of parametrization. In the case of complicated CAD models the regeneration domain can be hardly formulated in closed form, it can be only explored with the help of the CAD program. The statements above are highlighted on the simplest case in the next section.

\section{Regeneration domain and design space for two vari- ables}

The following simple example highlights the problem of having non-rectangular regeneration domain. Let us change the shape of an L-shaped body in one coordinate direction. The length parameters in vertical direction are allowed to change up to a mounting limit c (geometrical constraint). Moving dimensions can be selected in two ways as it is shown in Fig. 1 . According to the condition that topological changes are not allowed, the regeneration domains become in this case triangles (Fig.2).
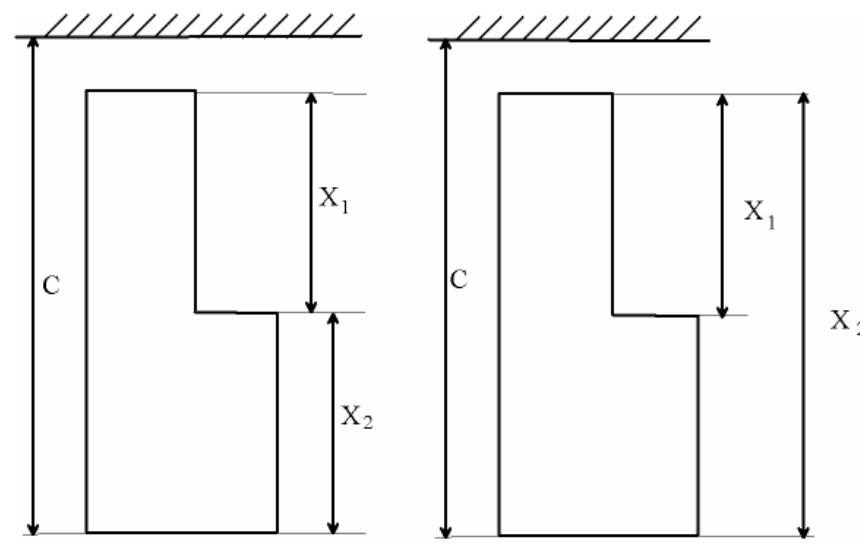

Fig. 1. L shaped body (left: size chain; right: base chain)

As it is mentioned before, design variables can be changed within constant bounds. If these limits are chosen on such a way that the optimization domain includes the whole regeneration domain (for chain sizes, the region bordered with dotted line in Fig. 2, left), there a great amount of solutions will be allowed that cannot be regenerated, therefore optimization may collapse. On the contrary, in order to have a feasible design, a rectangular area must be given inside of the triangle (bordered with dashed line, Fig. 2, left). In this case a reasonable part of possible solutions are excluded from the searching process.

All these problems can be avoided if the regeneration domain is transformed onto the unit square range of design variables $y_{1}, y_{2}$. Therefore design variables are also normalized, ensuring numerically more stable optimization.

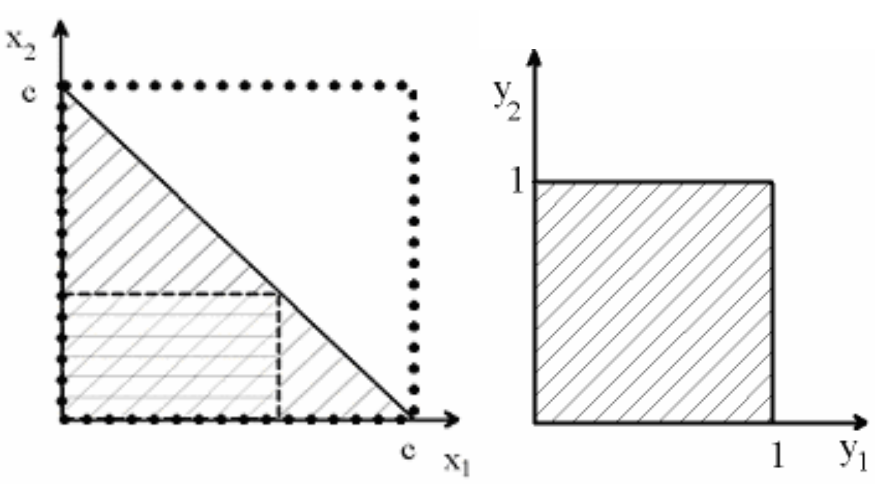

Fig. 2. left: regeneration domains for the $\mathrm{L}$ shaped body for size chain; right: design space)
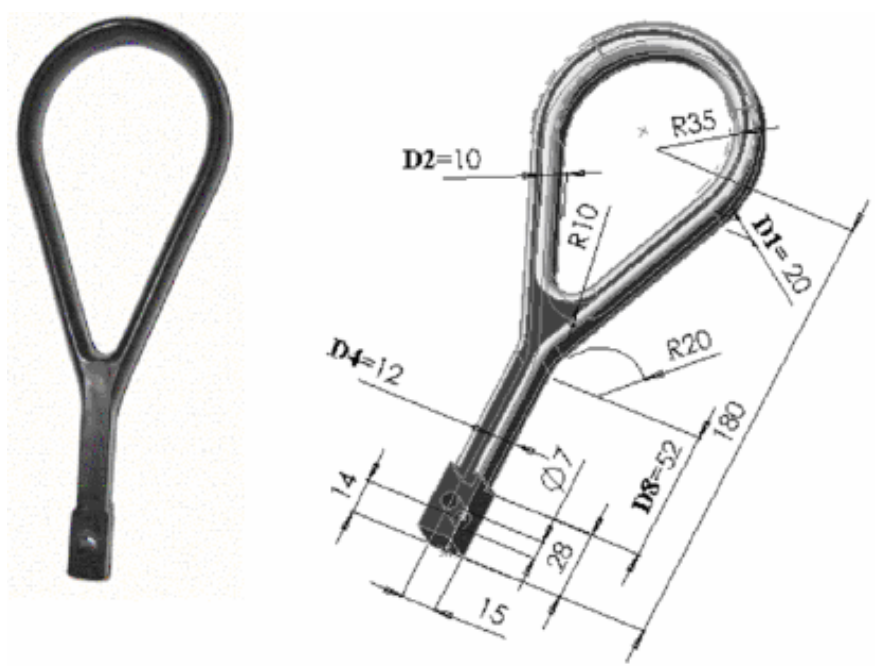

Fig. 3. left: Photo of the handhold; right: its CAD model

The transformation (the known Duffy transformation [8]) can be given as follows for size chain specification:

$$
\begin{aligned}
& x_{1}=c\left(y_{1}-y_{1} y_{2}\right) \\
& x_{2}=c y_{1} y_{2}
\end{aligned}
$$

On similar way, for base chain the regeneration domain is also a triangle and it can be mapped on more ways to the unit square, as previously:

$$
\begin{aligned}
& x_{1}=c y_{1} \\
& x_{2}=c\left(y_{1}+y_{2}\left(1-y_{1}\right)\right)
\end{aligned}
$$




\section{Optimization case study - handhold}

In order to illustrate the advantages of the proposed transformation method and show the finding of the regeneration domain and creation way of the transformation formulations, an optimization example of a handhold used in public transport vehicles was introduced, which was also studied with another optimization model in [9].

The photo and geometrical model of the handhold are shown in Fig. 3

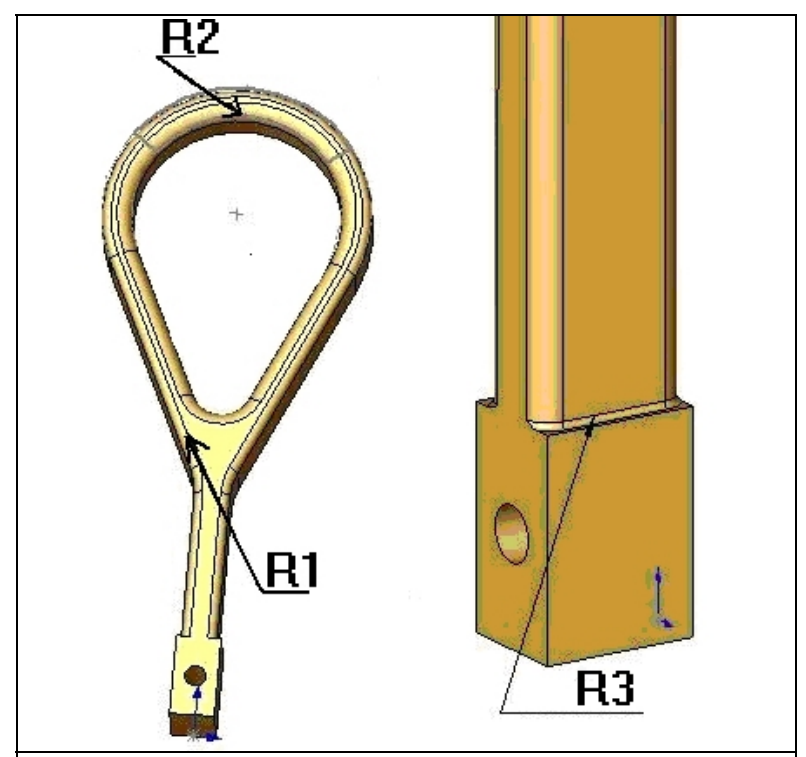

Fig. 4. The important filets of the geometry
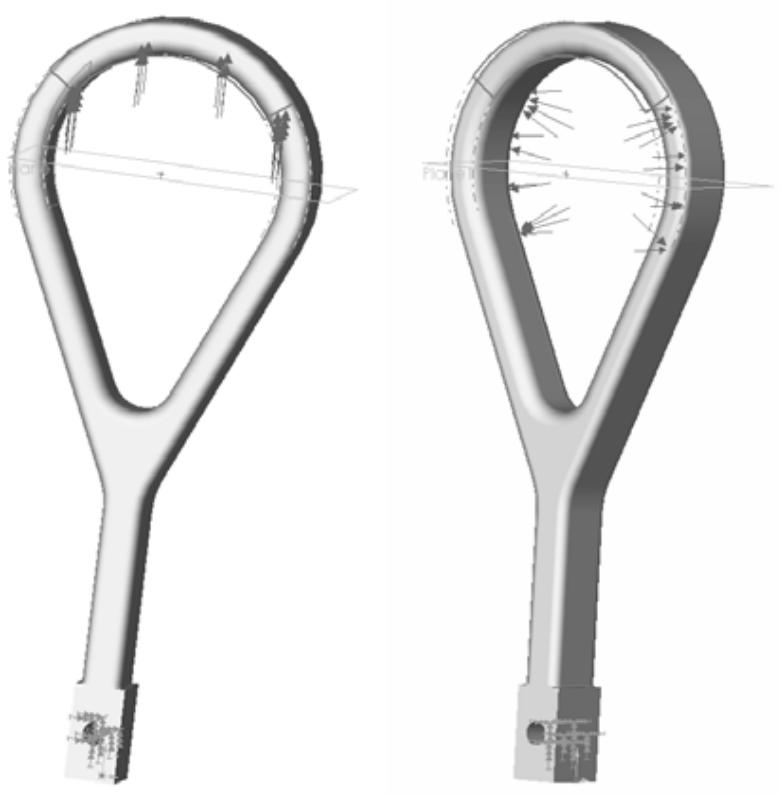

Fig. 5. Load cases

The initial geometrical model was generated by extrusion of a plane contour supplementing by 3 filets, where $\mathrm{R} 1$ is the radius of the fillet of the outer contour, $\mathrm{R} 2$ is the radius of the inner one and R3 is the radius of the fillet at the root of the arm (Fig. (4). These fillets are driven by the following equations in order to retain the proportion of the geometry:

$$
\begin{aligned}
R 1 & =\mathbf{D 4} / 4 \\
R 2 & =\mathbf{D} 1 / 4 \\
R 3 & =(15-\mathbf{D 4}) / 2
\end{aligned}
$$

where D1 and D4 are shown in Fig. 3, right.

By the structural model two load cases were taken into consideration (Fig. 5) : a $800 \mathrm{~N}$ tension in axial direction (load case 1) and a $30 \mathrm{~N}$ force on each of the two rounded surfaces of the handhold, actually representing a torsion (load case 2). (Although, theoretically, a situation can occur where the two loads have a joint impact on the optimum studied, in our assumption the load cases have a separate impact on the object examined so as to present the potentials of the optimization system.) The examined body was fixed on the hole in all degrees of freedom. In a closed environment of the smallest fillet (R3) geometry related mesh refinement was used for the better approximation of the stress concentration. In the FE model linear tetrahedron elements were used for the discretization of the structure, linear static elastic material properties of the polypropylene were taken into consideration.

In the optimization model the volume was chosen as an objective function, to be minimized. The displacement type optimization constraints were set to $3 \mathrm{~mm}$ in the first load case and to $7 \mathrm{~mm}$ in the second load case while the stress optimization constraint was set to $30 \mathrm{MPa}$ in both cases. Fig. 3 right shows the changing sizes: the size perpendicular to the handhold plane (D1); section width of the arched part (D2); width (D4) and length (D8) of the arm.

Using the given geometrical representation and structural model, let the variable dimensions be the design variables themtselves, and the optimization domain is given between $50 \%$ and $150 \%$ of the initial value for each variable. Unfortunately, the optimization loop break at the third iteration step as the geometry and the structural model is unrecoverable due to the resulted design point. Therefore other design variables must be chosen to overcome such problems.

If a mapping could be found between the regeneration domain of the problem and a normalized 4 dimensional rectangular space, the latter variables could be used as convenient design variables for the optimization. Therefore our task is first to explore the regeneration domain for the problem and then construct its mapping onto the unit cube.

\section{Regeneration domain}

By giving the regeneration domain in closed form, the dependency of the bounds for the variable dimensions must be found out. At the beginning an independent size must be found from the other variable dimensions. In general, thickness of the extrusion (D1) is a suitable choice.

There is no special condition for the lower limit (of course it should be positive); the upper limit will be $15 \mathrm{~mm}$ because of 
R3 must be positive, see Eq. [5:

$$
0<\mathbf{D} 4<15
$$

Thickness size D1 must be greater than $2 \mathrm{R} 1$ in order to avoid the change in the surface topology; the rest of changing dimensions does not exert any influence to the bounds of handhold thickness. Geometry regeneration is ensured according to equation (3) by the condition:

$$
\text { D4 } / 2=2 R 1<\mathbf{D 1}<\infty \text {, }
$$

which prohibits the disappearance of the surface between the two delimiting fillets. D1 should not be increased beyond all limits because the volume increases linearly with D1, therefore its value was limited to $25 \mathrm{~mm}$ :

$$
\text { D4 } / 2<\text { D1 }<25
$$

The remaining two variable sizes are included in the plane of the contour drawing.

By setting the upper limit of D2, it is considered that the volume increases for larger D2 values, therefore the maximum value was set to $30 \mathrm{~mm}$. The lower limit of D2 can also be derived from the conservation of the surface between the fillets of $\mathrm{R} 1$ and $\mathrm{R} 2$.

$$
0<\mathbf{D 2}-(R 1+R 2)
$$

Concerning Eqs. (3) and (4), the interval for D2 is the following:

$$
(\text { D1 }+ \text { D4) } / 4<\text { D2 }<30
$$

The lower limit of the arm length D8 is not critical, but it should naturally be positive. When specifying the upper limit, it should be taken into consideration that the length of the tangent line of circles R10 and R35 shown in Fig. 3 right, should not become zero. There are two ways to determine this correlation: either from the geometrical interrelations or experimentally with the sketcher that also offers approximation procedures for specification of the regeneration limit. In complicated cases it is difficult to specify the interaction of the sizes analytically, and the approximation of the regeneration limit with a given accuracy (in the example, the size change is phased to $1 \mathrm{~mm}$ ) is sufficient for most engineering problems. The approximation procedure was used in this case as well. The D4 dependence of the function to be found is small, therefore it is neglected, and D2 dependence can be substituted by a linear function:

$$
0<\text { D8 }<100-\text { D2 } \text {. }
$$

It should be mentioned, that the inequalities defining the regeneration domain are so constructed, that there is no cross linked dependency between the limits. The transformation then can be constructed due to appropriate arrangement of them and fitting the variables.

\section{Transformation}

The regeneration domain is given by inequalities (6), (8), (10) and (11) the transformation should be specified in form of equation $(12)$ :

$$
\mathbf{x}=F(\mathbf{y}),
$$

where $\mathbf{y}$ is the vector of the new design variables and $\mathbf{x}$ is the vector of the changing dimensions (D1,D2,D4,D8).

Matching $\mathrm{y}_{1}$ to D4, a simple scaling can be used. By the second inequality pair, first we have to scale to one with the upper limit, and than we order the lower bound to zero. Matching it to $y_{2}$ and substituting the first transformation equation for D4, the second transformation equation is given. On a similar way the other two equations can also be determined. It must be mentioned, that this technique works in case if the bounds are not cross dependent.

The correspondent relations of changing sizes and design variables are summarized:

$$
\begin{aligned}
& \mathbf{D 4}= 15 y_{1} \\
& \mathbf{D 1}=\frac{15}{2} y_{1}+25 y_{2}-\frac{15}{2} y_{1} y_{2} \\
& \mathbf{D 2}=y_{3}\left[30-\left(\frac{45}{2} y_{1}+25 y_{2}-\frac{15}{2 y_{1} y_{2}}\right) / 4\right]+ \\
&\left(\frac{45}{2} y_{1}+25 y_{2}-\frac{15}{2} y_{1} y_{2}\right) / 4 \\
& \mathbf{D 8}=y_{4}\left\{100-y_{3}\left[30-\left(\frac{45}{2} y_{1}+25 y_{2}-\frac{15}{2} y_{1} y_{2}\right) / 4\right]+\right. \\
&\left.\left(\frac{45}{2} y_{1}+25 y_{2}-\frac{15}{2} y_{1} y_{2}\right) / 4\right\}
\end{aligned}
$$

The initial values are summarized in Table 1 .

Tab. 1. Initial values of the optimization variables

\begin{tabular}{cc}
\hline Initial value of variable dimensions $[\mathrm{mm}]$ & Initial values of design variables \\
\hline $\mathbf{D} 4^{i n i}=12$ & $y_{1}^{i n i}=\frac{12}{15}$ \\
\hline $\mathbf{D 1}^{i n i}=20$ & $y_{2}^{i n i}=\frac{14}{19}$ \\
\hline $\mathbf{D} 2^{i n i}=10$ & $y_{3}^{i n i}=\frac{1}{11}$ \\
\hline $\mathbf{D 8}^{i n i}=52$ & $y_{4}^{i n i}=\frac{52}{100-\frac{1}{11}}$ \\
\hline
\end{tabular}

Applying this projection, optimization converged with no regeneration error in 28 steps. Results can be seen in Fig. 6

Optimization history can be followed on Fig. 7 It shows that as the volume is reduced considerably ( $33 \%$ ), stress constraint was activated in the bending load case, while the displacement constraint was activated the load case causing torsion stress.

\section{Conclusions}

It was pointed out that the use of variable dimensions itself as design variables are not satisfactory for successful optimization. A condition is given to ensure the recoverability of the optimization problem with the new design variables, independently from 

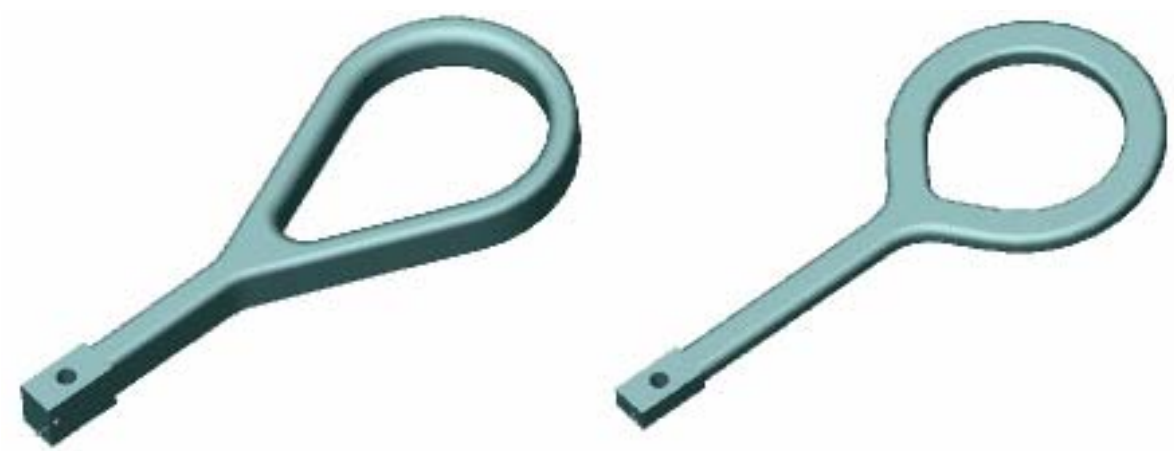

Fig. 6. Initial and optimal shape of handhold
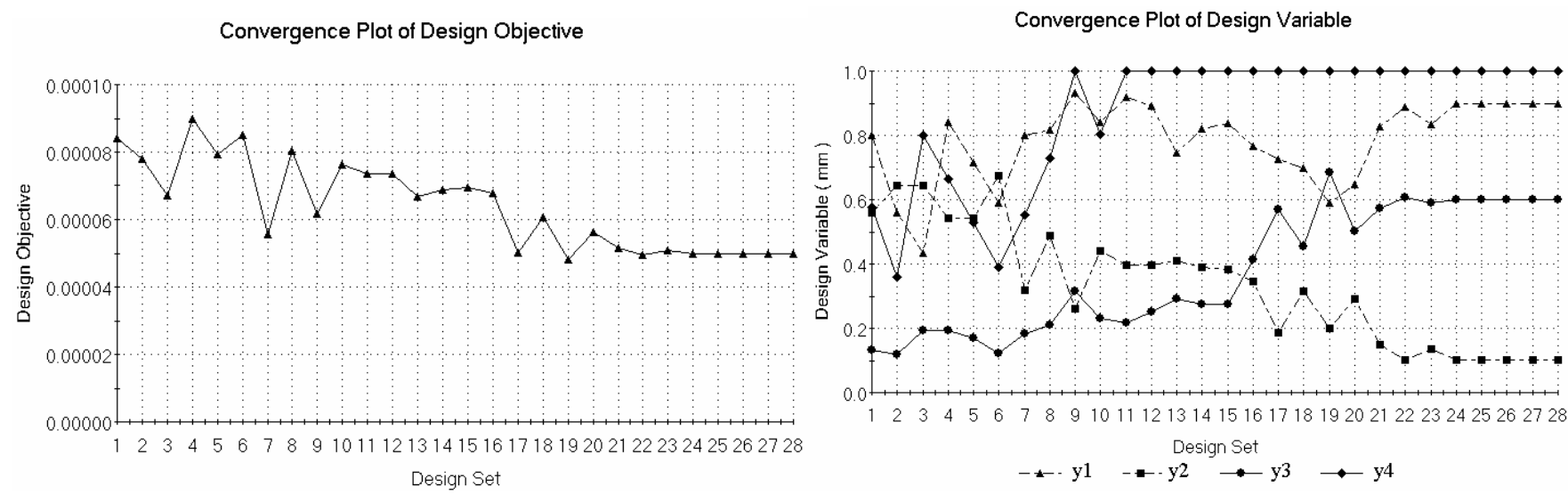

Fig. 7. History of the objective function and the design variables

the geometrical representation: if the surface topology remains the same as it was in the initial design, the problem can be built up. This topology condition determines the domain of feasible solutions. In the cases when this domain can be given or approximated in closed form, a transformation onto the unit cube of the same order is to be applied advantageously, which have to be derived individually for each problem by ordering the bounds of the domains to each other. It was shown in a case study on what manner the regeneration domain and the mapping can be given, which technique can be applied in other cases as well. The adaptability of the governing ideas are not restricted to the special case of the mentioned shape optimization but can be applied in general: it is no mean what kind of searching techniques or variables are used, the need of the mapping of the regeneration domain to the unit cube helps to perform a successful optimization without breaking or excluding valid solutions from the searching process.

\section{References}

1 Haftka RT, Kamat MP, Elements of Structural Optimization, Martinus Nijhoff Publishers, 1985.

2 Kirsch U, Optimum Structural Design, McGraw-Hill Book Company, 1981.

3 Arora JS, Optimization of Structural and Mechanical System, Word Scientific Publishing, 2007.

4 Yao TM, Choi KK, Three Dimensional Shape Optimal Design and Automatic Finite Element Regridding, International Journal for Numerical Methods in Engineering 28 (1989), no. 2, 369-384.

5 Vaidya A, Yu S, St Ville J, Nguyen DT, Rajan SD, Multiphysics CAD-
Based Design Optimization, Mechanics Based Design of Structures and Machines 34 (2006), no. 2, 157-180, DOI 10.1080/15397730600745807.

6 Hardee E, Chang KH, Tu J, Choi KK, Grindeanu I, Yu X, A CAD-based design parameterization for shape optimization of elastic solids, Advances in Engineering Software 30 (1999), no. 3, 185-199, DOI 10.1016/S09659978(98)00065-9.

7 Mathiak G, A Composition Strategy Applied to 3D Shape Optimization Using the Boundary Element Method and Nested Design Models, 1996. PhD. Thesis.

8 Duffy MG, Quadrature over a pyramid or cube of integrands with a singularity at a vertex, Siam. J. Numer. Anal. 19 (1982), no. 6, 1260-1262, DOI $10.1137 / 0719090$.

9 Erdős-Sélley Cs, Körtélyesi G, Shape Optimization and Modeling in an Integrated CAD/FEM Enviroment, Proceedings of 3rd Conference on Mechanical Engineering, Budapest University of Technology and Economics, Unknown Month May 30, pp. 649-653. 